\title{
Congenital herpes simplex virus infection among hospitalized infants in Poland
}

\author{
Michał Rząd ${ }^{1, A, C-F \oplus}$, Aneta Nitsch-Osuch ${ }^{1, A, E-F \oplus}$, Piotr Zbigniew Tyszko ${ }^{2,1, A, E-F} \oplus$, \\ Paweł Goryński ${ }^{3, A-B, E-F \oplus}$, Magdalena Bogdan ${ }^{1, A, E-F}{ }^{\oplus}, K^{2}$ atarzyna Lewtak ${ }^{1, A, E-F}{ }^{\oplus}$, \\ Krzysztof Kanecki ${ }^{1, A, C-F \odot ~}$ \\ ${ }^{1}$ Department of Social Medicine and Public Health, Medical University of Warsaw, Warsaw, Poland \\ ${ }^{2}$ Witold Chodźko Institute of Rural Health, Lublin, Poland \\ ${ }^{3}$ National Institute of Public Health NIH - National Research Institute, Warsaw, Poland \\ A - Research concept and design, B - Collection and/or assembly of data, C - Data analysis and interpretation, \\ $D$ - Writing the article, E-Critical revision of the article, F- Final approval of the article
}

Rząd M, Nitsch-Osuch A, Tyszko PZ, Goryński P, Bogdan M, Lewtak K, Kanecki K. Congenital herpes simplex virus infection among hospitalized infants in Poland. Ann Agric Environ Med. 2021; 28(4): 612-616. doi: 10.26444/aaem/142999

\begin{abstract}
Introduction and objective. Congenital herpes simplex virus infection (cHSV) is a rare entity and may pose a life-threatening disease for the newborns. Due to the public health and clinical importance of cHSV infection in infants, the recent data on this disease in Poland should be investigated. The aim of the study was to evaluate the incidence and other factors related to cHSV. Materials and method. The study is retrospective, population-based that utilised the hospital discharge records of cHSV patients. Data were obtained from National Institute of Public Health NIH - National Research Institute in Warsaw, Poland, covering the period 2014-2019.

Results. The study group consisted of 1,573 cHSV newborns (841 males and 732 females). Among this group, $70.1 \%$ were infants up to 90 days of age and 3.6\% were neonates up to 28 days of age. The mean and median age was 98 days (95\% Cl: 94.5-101.8, SD 74) and 70 days (IQR: 53-104), respectively. Based on the hospital registry the overall average cHSV incidence over the study period was estimated to be 69 per 100,000 live births. The number of cHSV infection fluctuated over the years 2014-2019 with a significant decreasing trend from 2015-2019 ( $\mathrm{P}<0.01)$. A significantly higher cHSV incidence was observed among patients from urban than rural areas of Poland (88 vs 40/100,000 live births; $P<0.001$ ).

Conclusions. CHSV incidence may be related to multifactorial conditions for the occurrence of this disease. Further studies on changes in the incidence of cHSV are needed.
\end{abstract}

\section{Key words}

epidemiology, HSV, TORCH, national register, congenital disease

\section{INTRODUCTION}

Congenital herpes simplex virus infection (cHSV) is a rare entity and may pose a life-threatening disease for the newborns. Herpes simplex virus (HSV) belongs to the alpha subfamily of the human herpes virus family with at least two distinct serotypes (HSV-1 and HSV-2).

According to information from World Health Organization, in 2016, an estimated 3.7 billion people under the age of 50, or $67 \%$ of the population, had HSV-1 infection. Estimated prevalence of the infection was highest in Africa (88\%) and lowest in the Americas (45\%). With regard to genital HSV-1 infection, an estimated 122 million - 192 million people aged 15-49-years had developed genital HSV-1 infection worldwide in 2016. Most genital HSV-1 infections are estimated to occur in the Americas, Europe and Western Pacific, where HSV-1 continues to be acquired into adulthood [1]. It was estimated that 23.9 million people $15-49$ years of age became infected with HSV-2 in 2016, an incidence of $0.6 \%$. Of these, 14.7 million were women and 9.2 million were men [2]. Additionally, in a large study from the USA, during 2015-2016, the prevalence of HSV-1 was 47.8\% and of HSV-2 was $11.9 \%$ among those aged 14-49 [3].

Address for correspondence: Krzysztof Kanecki, Department of Social Medicine and Public Health, Medical University, Oczki 3, 02-007 Warsaw, Poland E-mail: kanecki@mp.pl

Received: 20.07.2020; accepted: 22.09.2021; first published: 20.12.2021
HSV can be acquired by an infant during one of three periods: in utero $(5 \%)$, peripartum $(85 \%)$, or postnatal $(10 \%)$ [4]. HSV-2 is the cause of most genital herpes and is almost always sexually transmitted. HSV-1 is usually transmitted during childhood via non-sexual contacts. CHSV infection was reported to be associated with maternal age younger than 20 years, gestational age less than 32 weeks, and vaginal and emergency caesarean section deliveries [5]. No effective HSV vaccines are currently available [6]. Prenatal ultrasound abnormalities due to CHSV are rare, varied and non-specific. There is no clear role for foetal ultrasound in the routine management of women with primary or non-primary HSV infection in pregnancy. However, in foetuses with ultrasound abnormalities suggestive of congenital infection, HSV should still be considered [7].

Globally the overall rate of neonatal herpes was estimated to be about 10 cases per 100,000 live births. The most neonatal herpes cases occurred in Africa, due to high maternal HSV-2 infection and high birth rates. HSV-1 contributed more cases than HSV-2 in the Americas, Europe, and Western Pacific [8]. In the USA, in a prospective cohort of pregnant women enrolled between 1982 -1999, neonatal HSV infection rates per 100,000 live births were 54 among HSV-seronegative women, 26 among women who were HSV-1-seropositive only, and 22 among all HSV-2-seropositive women [9]. Moreover, neonatal HSV infections were reported to occur at a rate of 1 per 3,000 live births according to data collected over 7 years in Florida in the 
USA [10]. The incidence of neonatal HSV in the United States increased from 7.9 to 10 per 100,000 live births from 2003-2005 to 2012-2014 [11]. In another retrospective longitudinal cohort study using a database of neonates hospitalized with HSV infection from 2009 to 2015 , a corrected incidence rate of 4.5 per 10000 births was reported. The yearly disease incidence increased by $56 \%$, from 3.4 per 10,000 births in 2009 to 5.3 per 10,000 births in 2015 [12]. In Switzerland, between 2002-2008, the neonatal HSV infection incidence was reported to be 1.6 per 100,000 live births [13]. In The Netherlands, the neonatal herpes incidence was $4.8 / 100,000$ live births based, on survey data (2012-2015), and 3.4/100,000 based on data from the Perinatal Registry of The Netherlands (2012-2014) [14]. In England, in a population-based, observational cohort study which used national and regional hospitalization data from 1968-2016, calculating annual rates of hospital discharges coded with-and individuals aged younger than one month diagnosed with cHSV, in 2016, the discharge rate per 100,000 infant population was 17.6 for HSV, and compared with earlier years of the study, the discharge rate in 2016 was higher for HSV [5]. In France, neonatal herpes was reported to be rare, about 20 cases per year [15].

Women who have genital herpes before they become pregnant are at very low risk of transmitting HSV to their infants. The risk for neonatal herpes is the greatest when a mother acquires HSV infection for the first time in late pregnancy [1]. The risk of HSV transmission to a neonate born to a mother who acquires primary genital infection near the time of delivery is estimated to be $25 \%-60 \%$. In contrast, the risk to a neonate born to a mother shedding $\mathrm{HSV}$ as a result of reactivation of infection acquired during the first half of pregnancy or earlier, was reported to be less than $2 \%$ [16]. Seropositivity among pregnant women may be related to the risk of cHSV prenatally. In a study from Teheran, Iran, analyzing the prevalence of the herpes simplex virus (HSV) infection among pregnant women, umbilical cord blood samples from the newborns were analyzed for the presence of HSV DNA using PCR. HSV IgG and IgM antibodies were found in $97 \%$ and $2 \%$ of pregnant women, respectively. Of all the 100 cord blood samples, only 6 were positive for HSV DNA [17].

Raising the issue of cHSV is of paramount importance because of the impact of this disease on the developing bodies of infants. cHSV places the neonate at risk for mortality or long-term neurodevelopmental disability. HSV infection in newborn infants may be observed with the following manifestations: disseminated disease involving multiple organs, most prominently liver and lungs (25\%), localized central nervous system disease, with or without skin involvement $(30 \%)$, and disease limited to the skin, eyes, and/or mouth (45\%) [16]. Intrauterine HSV infections can lead to miscarriages, especially in the first half of pregnancy. In one study in 96 cases of stillbirth, the herpes viruses were found in the placenta and internal organs in 51 observations (53.1\%). Among them, HSV-1 were found in 16 observations (16.7\%), HSV-2, in 19 (19.7\%) [18].

An analysis of hospitalizations of patients with cHSV may provide a good estimate of its incidence in Poland. Hospitalization of infants due to cHSV lasting more than 7 days also generates high costs estimated at \$40,044 [19]. Due to the public health and clinical importance of cHSV infection in infants, the recent data on its epidemiology in Poland should be investigated.

\section{OBJECTIVE}

The aim of the study was to evaluate the incidence and other factors related to $\mathrm{cHSV}$.

\section{MATERIALS AND METHOD}

The study is population-based, retrospective analysis of hospital discharge records of infants with cHSV. Data were obtained from National Institute of Public Health NIH National Research Institute in Warsaw, Poland, covering the period 2014-2019. All hospitals in Poland, except psychiatric facilities, are legally required to send discharge data to the Institute. The data are anonymous and include information on hospitalizations with ICD-10 code diagnoses, dates of admission and discharge, gender, date of birth and place of residence. All hospitalization records with 'P35.2' as the primary of secondary ICD-10 code diagnoses were included in the study. cHSV may be described prenatally and postnatally, and the current study was based on the assumption that the diagnosis was concluded on widely accepted and used criteria, prenatal diagnosis during ultrasound screening and/or during diagnosis or a work-up for congenital malformations. In order to limit the impact of secondary hospitalizations on the incidence of cHSV, repeated hospitalizations that involved patients with the same date of birth, gender and code of residence were excluded. A retrospective study was performed of all patients who were hospitalized from 2014-2019. Information for the study was submitted to the local Bioethics Committee. According to the information obtained from the committee, in the case of retrospective and non-invasive research studies, the Bioethics Committee does not issue an opinion. cHSV often requires advanced differential diagnostic procedures and treatment during hospitalization; therefore, hospitalized cases may provide a good basis for estimating incidence. It was assumed that cHSV diagnoses were made in hospitals on the basis of the most current and widely used diagnostic criteria.

Statistical analysis. To perform the statistical analyses, Statistica (TIBCO Soft-ware Inc, Palo Alto, California, United States) [20] and WINPEPI [21] were used. The following statistical measures were computed: means, medians, $95 \%$ confidence intervals, and ranges for continuous variables, counts or percentages for categorical or nominal variables. Rates of hospitalizations related with cHSV infection were calculated as the estimated number of unique patients per 100,000 live births using data (national census) from the Central Statistical Office of Poland in Warsaw [22]. The mother's place of residence was assigned to the newborn using data also from from the Central Statistical Office of Poland. To assess trends, linear regression was used. When normality assumptions were not met, non-parametric tests (Chi-square, U Mann-Whitney) were applied. A two-sided p-value less than 0.05 was considered to be statistically significant.

\section{RESULTS}

During the study period, data of 2,391 hospitalizations of cHSV infants were analyzed. The study group consisted of 
1,573 cHSV newborns (841 males and 732 females). Among this group $70.1 \%$ were infants up to 90 days of age and $3.6 \%$ were neonates up to 28 days of age. The mean and median age was 98 days (95\% CI: 94.5-101.8, SD 74) and 70 days (IQR: 53-104), respectively. Based on the hospital registry the overall average cHSV incidence over the study period was estimated to be 69 per 100,000 live births. The number of cHSV infection fluctuated over the years 2014-2019, with a significant decreasing trend from 2015-2019 $(P<0.01)$ (Fig. 2). A significantly higher cHSV incidence was observed among patients from urban than rural areas of Poland (88 vs 40 per $100,000$ live births, $\mathrm{P}<0.001)$. Distribution of age of diagnosis during first-time hospitalizations is presented in Figure 1. Annual number of cHSV cases is shown in Figure 2. No deaths were reported during the analyzed period.

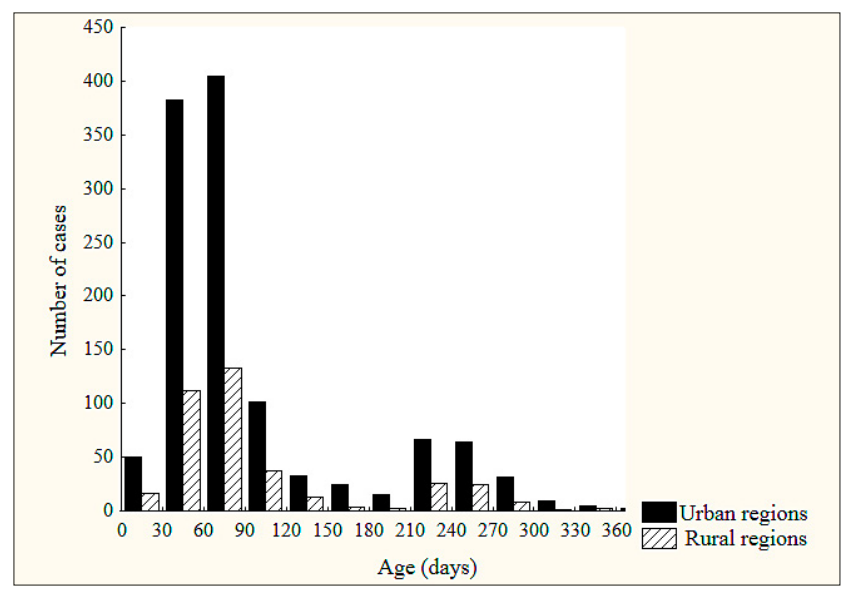

Figure 1. Age distribution of cHSV cases by urban and rural regions of Poland 2014-2019

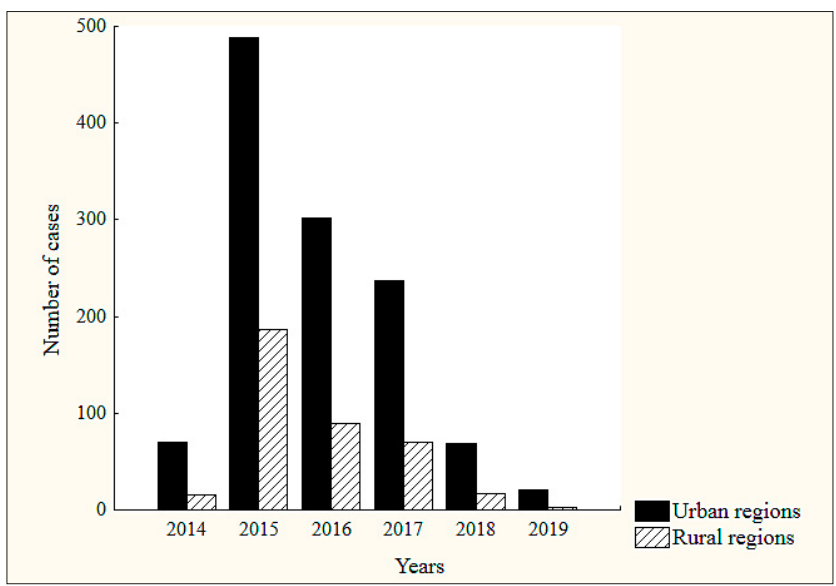

Figure 2. Number of cHSV cases by urban and rural regions of Poland by year, 2014-2019

\section{DISCUSSION}

Based on hospital registry, the average cHSV incidenc in Poland was estimated to be 69 per 100,000 live births. In a study from England based on hospitalisation data of hospital discharges and unique individuals aged less than 1 month, cHSV discharge rate was reported to be 17.6 per 100,000 infant population in 2016. Additionally, compared with the discharge rates in the 1980s and 1990s, these data represented a 7-fold increase [5]. A retrospective study conducted in the United States based on births from 2009-2015 reported a corrected incidence rate of 4.5 per 10,000 births. Additionally, the yearly disease incidence in this study also increased by $56 \%$, from 3.4 per 10,000 births in 2009 to 5.3 per 10,00 births in 2015 [12]. Estimates of cHSV depending on different World Health Organization (WHO) regions also indicate a lower incidence of this disease for the whole world and for Europe [8]. Such a high incidence of cHSV in Poland highlights the need for public health policies to increase awareness and education about prevention methods among women and men living in Poland.

The observed significantly decreasing trend in the number of cHSV cases in Poland in 2015-2019 may be related both to changes in individual behaviours or healthcare organization in Poland. Changes in cHSV incidence were also reported in other regions. HSV-1 epidemiology was reported to undergo a remarkable transition in the USA, with less exposure in childhood and more in adulthood, and less oral but more genital acquisition. HSV-1 will persist as a widely prevalent infection, with an ever-increasing genital disease burden [23].

The age distribution of the study group in the current study shows first, a peak in the first 60-90 days of life, and a second peak after half year (Fig. 1). It can be presumed that the first peak may be related to a gradual disappearance of maternal antibodies from transplacental transport and mother's milk. Another possible mechanism is postnatal infections after contact with persons with the orolabial form of the disease. The second peak may suggest an asymptomatic or oligosymptomatic form of cHSV infection and gradual disease development requiring hospitalization after many months. On the other hand, the presented results may indicate that the disease may take different forms which may be due to many other factors. Further studies on the epidemiology of this disease are needed.

In the current study, differences between rural and urban residents were observed: a significantly higher cHSV incidence was observed in urban than rural areas of Poland. Although sexual behaviours are of great importance in the spread of cHSV, place of residence as a factor may play an important role in cHSV incidence differences observed in this study. Other studies may also suggest this relationship. A study conducted among pregnant women in rural regions of India have shown a lower seroprevalence of HSV-2 relative to studies in a given region [24]. Other studies originating in China have also shown that the seroprevalence of HSV2 was higher in individuals from the eastern region and urban areas of Shandong than those from other regions [25]. The other large epidemiological study on the occurrence of HSV in the Polish population covering 4 regions reported geographical place of residence as a significant risk factor for HSV-2 seropositivity [26].

During the observed period, no deaths due to $\mathrm{CHSV}$ were reported, despite the relatively high percentage of deaths due to $\mathrm{CHSV}$ reported in other studies. In a retrospective study that characterized the clinical course of neonates who died with herpes simplex virus infection from 2001-2011, the casefatality rate was reported to be $26 \%$ [27]. In another study from Australia in a prospective national active surveillance from 1997-o 2011, the overall mortality was $18.8 \%$ [28]. In a study based on neonatal HSV-related deaths in New York 
City, it was highlighted that the increasing neonatal HSVrelated death rate may reflect increases in neonatal herpes incidence. An increasing number of pregnant women who have never had HSV-1 are therefore at risk of acquiring infection during pregnancy and transmitting it to their infant [29]. In one study from The Netherlands, the mortality rate was reported to be $23 \%$. In this study, the incidence of neonatal herpes increased slightly over time, as did the prevalence of genital HSV infection among pregnant women [14]. A high seroprevalence of HSV-1 may also be related to the risk of acquiring infection during pregnancy and transmission to the infant. In a large survey it was reported that a notably high seroprevalence of HSV-1, even among young female adolescents $15-19$ years of age (80\%) was observed. However, in Poland, HSV-2 seropositivity was under $12 \%$ in all age groups surveyed, which tended to be among the lowest overall HSV-2 seropositivity rates reported in Europe [26]. In another study analyzing seroprevalences of antibodies against 10 TORCH pathogens, reported the prevalence of HSV-1 in Poland at $85.7 \%$, and HSV-2 for Poland at $4.1 \%$ [30].

The lack of deaths observed in the current study may also result from both the clear recommendations of the Polish Gynaecological Society regarding HSV infection and the management of pregnant women, as well as increasingly better standards of perinatal care in Poland [31]. Neonatal HSV disease, although rare, is a serious, life-threatening condition, and decreasing delay in the initiation of acyclovir treatment may improve outcomes [32].

The presented database-based retrospective study, despite its many advantages, also has its limitations. During analysis, the hospitalization register was mainly relied on. Based on data from hospital records, it was possible to estimate the current cHSV incidence. During the study itself, the correctness of diagnoses and the precision of reporting were not verified, which may be a factor disturbing the results. Furthermore, only inpatient hospitalization discharge records were included in the database, which means that part of outpatients with cHSV were excluded from the study. In 5 cases in this study, the place of residence was not known. Another limitation is related to the date of the first hospitalization, which might not necessarily be the date of the first cHSV diagnosis. Because of this inaccuracy, the incident cases might have been imprecisely estimated; however, a long observation period and a large size of the data sample obtained from the national register of hospital morbidity can minimize this inaccuracy.

\section{CONCLUSIONS}

In Poland in 2014-2019, the cHSV incidence based on infants hospitalization fluctuated and was more often observed among infants from urban than rural areas, suggesting multifactorial conditions for the occurrence of this disease.

\section{REFERENCES}

1. https://www.who.int/news-room/fact-sheets/detail/herpes-simplexvirus (access: 2021.01.31)

2. James C, Harfouche M, Welton NJ, et al. Herpes simplex virus: global infection prevalence and incidence estimates, 2016. Bull World Health Organ. 2020; 98(5): 315-329. https://doi.org/10.2471/BLT.19.237149
3. McQuillan M, Kruszon-Moran D, Flagg EW, et al. Prevalence of Herpes Simplex Virus Type 1 and Type 2 in Persons Aged 14-49: United States, 2015-2016. NCHS Data Brief. 2018; (304): 1-8.

4. Bhatta AK, Keyal U, Liu Y, et al. Vertical transmission of herpes simplex virus: an update. J Dtsch Dermatol Ges J Ger Soc Dermatol JDDG. 2018; 16(6): 685-692. https://doi.org/10.1111/ddg.13529

5. Kadambari S, Pollard AJ, Goldacre MJ, et al. Congenital viral infections in England over five decades: a population-based observational study. Lancet Infect Dis. 2020; 20(2): 220-229. https://doi.org/10.1016/S14733099(19)30416-5

6. Xingli Xu, Ying Zhang, Quihan Li. Characteristics of herpes simplex virus infection and pathogenesis suggest a strategy for vaccine development. Rev Med Virol. 2019; 29(4): e2054. https://doi.org/10.1002/ rmv. 2054

7. Fa F, Laup L, Mandelbrot L, et al. Fetal and neonatal abnormalities due to congenital herpes simplex virus infection: a literature review. Prenat Diagn. 2020; 40(4): 408-14. https://doi.org/10.1002/pd.5587

8. Looker KJ, Magaret AS, May MT, et al. First estimates of the global and regional incidence of neonatal herpes infection. Lancet Glob Health. 2017; 5(3): e300-9. https://doi.org/10.1016/S2214-109X(16)30362-X

9. Brown Za, Wald A, Morrow Ra, et al. Effect of serologic status and cesarean delivery on transmission rates of herpes simplex virus from mother to infant. JAMA. 2003; 289(2): 203-209. https://doi.org/10.1001/ jama.289.2.203

10. Matthias J, du Bernard S, Schillinger JA, et al. Estimating neonatal herpes simplex virus incidence and mortality using capture-recapture, Florida. Clin Infect Dis Off Publ Infect Dis Soc Am. 2020; ciaa727. https://doi.org/10.1093/cid/ciaa727

11. Donda K, Sharma M, Amponsah JK, et al. Trends in the incidence, mortality, and cost of neonatal herpes simplex virus hospitalizations in the United States from 2003 to 2014. J Perinatol. 2019; 39(5): 697-707. https://doi.org/10.1038/s41372-019-0352-7

12. Mahant S, Hall M, Schondelmeyer AC, Berry JG, Kimberlin DW, Shah SS, et al. Neonatal Herpes Simplex Virus Infection Among MedicaidEnrolled Children: 2009-2015. Pediatrics. 2019 Apr; 143(4): e20183233.

13. Pascual A, Moessinger A, Gerber S, et al. Swiss Paediatric Surveillance Unit (SPSU). Neonatal herpes simplex virus infections in Switzerland: results of a 6-year national prospective surveillance study. Clin Microbiol Infect Off Publ Eur Soc Clin Microbiol Infect Dis. 2011; 17(12): 1907-10. https://doi.org/10.1111/j.1469-0691.2011.03641.x

14. van Oeffelen L, Biekram M, Poeran J, et al. Update on Neonatal Herpes Simplex Epidemiology in the Netherlands: A Health Problem of Increasing Concern? Pediatr Infect Dis J. 2018; 37(8): 806-13. https:// doi.org/10.1097/INF.0000000000001905

15. Renesme L. [Neonatal herpes: Epidemiology, clinical manifestations and management. Guidelines for clinical practice from the French College of Gynecologists and Obstetricians (CNGOF)]. Gynecol Obstet Fertil Senol. 2017 Dec; 45(12): 691-704. French. https://doi.org/10.1016/j. gofs.2017.10.005

16. Fernandes ND, Arya K, Ward R. Congenital Herpes Simplex. In: StatPearls [Internet]. Treasure Island (FL): StatPearls Publishing; January 11, 2021. http://www.ncbi.nlm.nih.gov/books/nbk507897/ (access 2020.11.30).

17. Tavakoli A, Monavari SH, Bokharaei-Salim F, et al. Asymptomatic Herpes Simplex Virus Infection in Iranian Mothers and Their Newborns. Fetal Pediatr Pathol. 2017; 36(1): 27-32. https://doi.org/1 $0.1080 / 15513815.2016 .1229368$

18. Glukhovets BI, Glukhovets NG, Belitchenko NV, et al. Immunofluorescence diagnosis of the herpesvirus stillborn infection. Vopr Virusol. 2016; 61(5): 219-221.

19. Owusu-Edusei K, Flagg EW, Gift TL. Hospitalization cost per case of neonatal herpes simplex virus infection from claims data. J Pediatr Nurs. 2015; 30(2): 346-352. https://doi.org/10.1016/j.pedn.2014.08.004.

20. TIBCO Software Inc. 2017. Statistica (data analysis software system), version 13. http://www.tibco.com, Palo Alto, CA, USA.

21. Abramson JH WINPEPI updated: computer programs for epidemiologists, and their teaching potential. Epidemiol Persp. 2011; 8: 1.

22. Statistics Poland. Available from: https://demografia.stat.gov.pl (access 2021.09.22)

23. Ayoub HH, Chemaitelly H, Abu-Raddad LJ. Characterizing the transitioning epidemiology of herpes simplex virus type 1 in the USA: model-based predictions. BMC Med. 2019; 17(1): 57. https://doi. org/10.1186/s12916-019-1285-x

24. Bochner AF, Madhivanan P, Niranjankumar B, et al. The Epidemiology of Herpes Simplex Virus Type-2 Infection among Pregnant Women in Rural Mysore Taluk, India. J Sex Transm Dis. 2013; 2013: 750415. https://doi.org/10.1155/2013/750415 
25. Huai P, Li F, Li Z, et al. Seroprevalence and associated factors of HSV2 infection among general population in Shandong Province, China. BMC Infect Dis. 2019; 19(1): 382. https://doi.org/10.1186/s12879-0193995-2

26. Smith JS, Rosinska M, Trzcinska A, et al. Type specific seroprevalence of HSV-1 and HSV-2 in four geographical regions of Poland. Sex Transm Infect. 2006; 82(2): 159-63. https://doi.org/10.1136/sti.2005.015446

27. Lopez-Medina E, Cantey JB, Sánchez PJ. The mortality of neonatal herpes simplex virus infection. J Pediatr. 2015; 166(6): 1529-1532.e1. https://doi.org/10.1016/j.jpeds.2015.03.004

28. Jones CA, Raynes-Greenow C, Isaacs D, on behalf of the Neonatal HSV Study Investigators and Contributors to the Australian Paediatric Surveillance Unit. Population-Based Surveillance of Neonatal Herpes Simplex Virus Infection in Australia, 1997-2011. Clin Infect Dis. 2014; 59(4): 525-531. https://doi.org/10.1093/cid/ciu381

29. Sampath A, Maduro G, Schillinger JA. Infant Deaths Due To Herpes Simplex Virus, Congenital Syphilis, and HIV in New York City.
Pediatrics. 2016; 137(4): e20152387. https://doi.org/10.1542/peds.20152387

30. Warnecke JM, Pollmann M, Borchardt-Lohölter V, et al. Seroprevalences of antibodies against ToRCH infectious pathogens in women of childbearing age residing in Brazil, Mexico, Germany, Poland, Turkey and China. Epidemiol Infect. 2020; 148: e271. https://doi.org/10.1017/ S0950268820002629

31. Karowicz-Bilińska A, Kurowska E, Nowak-Markwitz E, et al. Polish Gynecological Society. Rekomendacje Polskiego Towarzystwa Ginekologicznego dotyczące postępowania w przypadku zakazenia wirusem HSV w położnictwie [Management of Herpes Simplex Virus infection in obstetrics. Recommendations of the Polish Gynecological Society]. Ginekol Pol. 2015; 86(9): 715-717. Polish. http://doi:10.17772/ $\mathrm{gp} / 59226$

32. Harris JB, Holmes AP. Neonatal Herpes Simplex Viral Infections and Acyclovir: An Update. J Pediatr Pharmacol Ther. 2017; 22(2): 88-93. https://doi.org/10.5863/1551-6776-22.2.88 